\title{
Correction note to: View on a mechanistic model of Chlorella vulgaris in incubated shake flasks
}

\author{
Fabian Kuhfuß ${ }^{1}$ (D) $\cdot$ Veronika Gassenmeier ${ }^{1}$ - Sahar Deppe ${ }^{2} \cdot$ George $_{\text {Ifrim }}^{3}$ - Tanja Hernández Rodríguez ${ }^{1}$. \\ Björn Frahm ${ }^{1}$
}

Published online: 27 January 2022

(c) Springer-Verlag GmbH Germany, part of Springer Nature 2022

\section{Bioprocess and Biosystems \\ Engineeringhttps://doi.org/10.1007/s00449- 021-02627-2}

We noticed that the article contains the following errors, which have been corrected:

- In equation number 8 a the variable $X$ has been missing at the end. (This equation also appears in the graphical abstract. Article and graphical abstract have been updated.)

- The unit of biomass concentration $X$ has been corrected in Table 2 and in the list of abbreviations ( $\mathrm{g} / \mathrm{L}$ instead of $\mathrm{g} /(\mathrm{L} \mathrm{h}))$.
- A typo in the second affiliation has been corrected.

- The online resource document has been updated. (The previous document was not the final version.)

This has no impact on the conclusions of the article. The authors would like to apologize for any inconvenience caused.

Publisher's Note Springer Nature remains neutral with regard to jurisdictional claims in published maps and institutional affiliations.

The original article can be found online at https://doi.org/10.1007/ s00449-021-02627-2.

Björn Frahm

bjoern.frahm@th-owl.de

1 Biotechnology and Bioprocess Engineering, Ostwestfalen-

Lippe University of Applied Sciences and Arts, Campusallee

12, Lemgo, Germany

2 Frauenhofer IOSB-INA, Lemgo, Germany

3 "Dunarea de Jos" University of Galati, Galati, Romania 